\title{
Diagnóstico bioquímico positivo en pacientes con sospecha clínica de Niemann Pick C.
}

\author{
Positive Biochemical Diagnosis in Patients with Clinical Suspicion of Niemann \\ Pick C.
}

\author{
Liz Carolina Pardo Echeverría ${ }^{\text {** }}$ \\ Leopoldo Antonio Arrieta Violet ${ }^{\mathrm{b}}$ \\ Fernanda Timm Seabra Souza ${ }^{c}$
}

Recepción: 18-ene-2016

Aceptación: 05-may-2016

\section{Resumen}

Se realizó diagnóstico bioquímico en cultivos de fibroblastos, provenientes de biopsias de piel de 73 pacientes candidatos clínicamente de padecer la enfermedad neurodegenerativa de herencia autosómica recesiva de Niemann Pick C, remitidos por los servicios de neurología y genética del país. Mediante análisis cualitativo de intensidad de fluorescencia perinuclear por tinción de Filipin, se reportaron 23 pacientes positivos (11 Mujeres/12 Hombres). La prueba t permitió inferir que no existen diferencias estadísticamente significativas en relación a la edad ( 1 - >45 años) y el género de los pacientes.

Palabras clave: Enfermedad de Niemann Pick C, depósito lisosomal, diagnóstico bioquímico, Lipoproteínas de baja densidad-LDL, lisis celular, Tinción de Filipin, Transporte intracelular de lípidos.

\begin{abstract}
Filipin staining test was made from cultured fibroblasts from skin biopsies from 73 patients clinically candidates of developing the neurodegenerative disease autosomal recessive Niemann Pick C, referred by the neurology and genetics in the country. Qualitative analysis by perinuclear fluorescence intensity, the positive biochemical diagnosis was identified in 23 patients. The $t$ test allowed inferring that not statistically significant differences in relation to age (1->45 years) and gender of patients.
\end{abstract}

Key words: Cell lysis, Filipin staining test, Lipid trafficking intracelular, low density lipoproteins-LDL, Lysosomal storage, Niemann Pick type C disease.

\footnotetext{
aMaestría en Ciencias Biológicas, Universidad Pedagógica y Tecnológica de Colombia, Grupo de Estudios en Genética y Biología Molecular-GEBIOMOL, Centro de Investigación en Genética Humana y Reproductiva-GENETIX.

*Autor de correspondencia: lizcapar@yahoo.com.mx

${ }^{b}$ Universidad Pedagógica y Tecnológica de Colombia, Grupo de Estudios en Genética y Biología Molecular, GEBIMOL.

${ }^{\mathrm{c}}$ Universidad Federal de Rio Grande de Sur, Instituto de Ciencias Básicas de Salud, Departamento de Bioquímica. Hospital de Clínicas de Porto Alegre, Servicio de Genética Médica.
} 


\section{Introducción}

Niemann Pick C (NPC) es una enfermedad neurodegenerativa con un patrón de herencia autosómica recesiva, caracterizada por deficiencia en el transporte intracelular de colesterol no esterificado, producido por la acumulación de glucoesfingolípidos en los lisosomas [1, 2, 3]. Su incidencia es de 1:120000 nacidos vivos. La expresión de la enfermedad ha sido asociada a las mutaciones en los genes NPC1 y NPC2 localizados en los cromosomas 18 y 14, loci (q11-q12) y (q24.3) respectivamente [4]. Las proteínas que codifican estos genes están involucradas en la exportación adecuada de colesterol y es posible que actúen de forma conjunta para regular el transporte lipídico intracelular y su homeostasis [5] (tabla 1). Mutaciones en estos genes generan disfuncionalidad, lo que altera el transporte lipídico y conduce a la acumulación de colesterol en los lisosomas [6].

\subsection{Metodología}

Medios de cultivo y suplementos. Minimum Essential Medium Eagle (MEM, Gibco-Invitrogen): suplementado con el $20 \%$ de Suero Fetal Bovino, Antibiótico-Antimicótico (Anti-Anti, GibcoInvitrogen), L-Glutamina, Lipoproteína -LDL(L7914, 50mg/ml Sigma) y Filipin III from Streptomyces filipinensis (Sigma).

Aspectos éticos. Al momento de la toma de muestra biológica se diligenció el consentimiento informado de todos los pacientes o sus tutores responsables.

Cultivos de fibroblastos y condiciones in vitro. Se evaluaron 73 pacientes a partir de biopsias de piel de 3-6 mm (Epidermis); tomadas por personal especializado. Se establecieron por duplicado los cultivos de fibroblastos en monocapa a $37^{\circ} \mathrm{C}$ en presencia de Humedad relativa de $95 \%$ y $5 \%$ de concentración de $\mathrm{CO}_{2}$, con un tiempo máximo de procesamiento de 24 horas después de la recolección de la muestra biológica. Se establecieron cultivos controles provenientes de pacientes con resultado previo negativo, con las mismas condiciones para comparar morfología y monitorear la prueba bioquímica empleada.

\section{Esterificación de lipoproteínas de baja densidad} -LDL. Se aplicó protocolo descrito por Vanier y colaboradores $[9,10]$, se estimulan los fibroblastos de cultivos celulares con una confluencia $>80 \%$ con medio libre de lipoproteína y adición $20 \mu 1$ a una concentración de $50 \mathrm{mg} / \mathrm{ml}$ de LDL durante 24 horas con posterior tinción con Filipin III.

Microscopía fluorescente. Las emisiones fluorescentes fueron capturadas con objetivo de $20 \mathrm{X}$ y 100X con lámpara de mercurio de 100 watt, con microscopio Olympus BX51 y filtro DAPI (350-450 nm) con registro de microfotografías. Como controles de lectura se utilizaron láminas de pacientes NPC facilitados por el Laboratorios de Clínicas (Porto Alegre-Brasil), Universidad de la Plata (Argentina) y Gillet-Mérieux et Neurogenétique Moleculaire, (Lyon-Francia). Cada resultado fue informado al médico tratante quien interpretará e informará al paciente sobre los hallazgos de la prueba bioquímica.

Tratamiento Estadístico. Bajo los valores de la prueba $t$ se comparó la distribución y significancia estadística de los pacientes con diagnóstico bioquímico positivo con relación a los datos demográficos de edad y género.

El diagnóstico bioquímico requiere el cultivo de fibroblastos para detectar el depósito de colesterol no esterificado en vesículas perinucleares, se considera que el $80-85 \%$ de los pacientes afectados presentan intensa fluorescencia $[9,10]$. Sin embargo, en Colombia es una patología subdiagnósticada por su heterogeneidad clínica, ausencia de registros de la población afectada y estandarización de pruebas de confirmación diagnóstica, por lo tanto, este diagnóstico bioquímico mediante tinción de Filipin permitió identificar la intensidad de fluorescencia perinuclear en tres patrones bioquímicos: positivo (presencia de vesículas perinucleares con intensa fluorescencia), negativo (ausencia de intensidad de la fluorescencia a nivel perinuclear) y fenotipo bioquímico variante (Intensidad de fluorescencia menos pronunciada), encontrados en los pacientes remitidos por los servicios de neurología y genética, con sospecha clínica y alto riesgo de padecer la enfermedad de NPC; para este estudio, se reportan únicamente los pacientes con el patrón clásico positivo.

Los datos obtenidos en ésta investigación, aportaron información de una población colombiana con alta sospecha de padecer la enfermedad de NPC y se generó una bases de datos que contribuya al planteamiento de otras investigaciones dirigidas a la implementación de pruebas alternativas de diagnóstico temprano. Al mismo tiempo, el personal médico dispondrá de una prueba bioquímica que permita el acercamiento al diagnóstico clínico y manejo terapéutico que modifica la historia natural de la enfermedad. 
Tabla 1. Localización cromosómica, mutaciones en los genes NPC1 y NPC2 e impacto celular en pacientes con NPC [5].

\begin{tabular}{|c|c|c|}
\hline Gen-proteína & $\begin{array}{l}\text { gen NPC1 } \\
\text { (proteína NPC1) }\end{array}$ & $\begin{array}{l}\text { gen NPC2 } \\
\text { (proteína NPC2) }\end{array}$ \\
\hline Gen-locus & & \\
\hline Proteína/Exones & 1278aa/25 exones & 132aa/5 exones \\
\hline Mutaciones (\%) & $\begin{array}{l}85-95 \%: \\
332 \text { mutaciones reportadas }\end{array}$ & $\begin{array}{l}4-5 \%: \\
20 \text { mutaciones reportadas }\end{array}$ \\
\hline $\begin{array}{l}\text { Disfuncionaliad } \\
\text { parcial de organelos }\end{array}$ & $\begin{array}{l}\text { Endosomas tardíos } \\
\text { Retículo endoplasmático } \\
\text { Complejo de Golgi } \\
\text { Membrana plasmática }\end{array}$ & $\begin{array}{l}\text { Endosomas tardíos } \\
\text { Lisosomas }\end{array}$ \\
\hline Codifica & $\begin{array}{l}\text { Una glicoproteína de membrana } \\
\text { endosómica }\end{array}$ & $\begin{array}{l}\text { Pequeña proteína lisoso- } \\
\text { mal }\end{array}$ \\
\hline Función & $\begin{array}{l}\text { Transporte intracelular, concentra- } \\
\text { ción y distribución del colesterol }\end{array}$ & $\begin{array}{l}\text { Proteína de unión a coleste- } \\
\text { rol }\end{array}$ \\
\hline $\begin{array}{l}\text { Implicación } \\
\text { biológica }\end{array}$ & $\begin{array}{l}\text { Acumulación de esfingosina, } \\
\text { esfingolípidos y colesterol } \\
\text { Alteración en la homeostasis del } \\
\text { calcio }\end{array}$ & $\begin{array}{l}\text { Alteración en la homeosta- } \\
\text { sis de esteroles. }\end{array}$ \\
\hline
\end{tabular}

Tabla 2. Manifestaciones Sistémicas y neurológicas según edad de aparición [7].

\begin{tabular}{|c|c|c|}
\hline Edad de inicio & $\begin{array}{l}\text { Manifestaciones sistemá- } \\
\text { ticas }\end{array}$ & Manifestaciones neurológicas \\
\hline $\begin{array}{l}\text { Pre-perinatal } \\
>3 \text { meses }\end{array}$ & $\begin{array}{l}\text { Hidrops fetal } \\
\text { Hepatoesplenomegalia } \\
\text { Ascitis fetal } \\
\text { Coleostasis prolongada } \\
\text { Infiltración pulmonar } \\
\text { Insuficiencia respiratoria } \\
\text { y/o hepática }\end{array}$ & No suele observarse \\
\hline $\begin{array}{l}\text { Infantil precoz } \\
3 \text { meses a }>2 \text { años }\end{array}$ & Hepatoesplenomegalia & $\begin{array}{l}\text { Retraso del desarrollo motriz } \\
\text { Hipotonía central } \\
\text { Hipoacusia } \\
\text { *PSMV (no detectado) }\end{array}$ \\
\hline $\begin{array}{l}\text { Infantil tardío } \\
2 \mathrm{a}<6 \text { años }\end{array}$ & Organomegalia & $\begin{array}{l}\text { Caídas frecuentes } \\
\text { Ataxia, distonía, disfagia, disartria. } \\
\text { Hipotonía central } \\
\text { Perdida de la audición } \\
\text { Convulsiones (parciales o generalizadas) } \\
\text { Cataplexia } \\
\text { *PSMV (habitualmente presente) }\end{array}$ \\
\hline $\begin{array}{l}\text { Juvenil (clásico) } \\
6 \text { a } 15 \text { años }\end{array}$ & $\begin{array}{l}\text { Organomegalia } \\
\text { (No siempre presente) }\end{array}$ & $\begin{array}{l}\text { Fracaso escolar } \\
\text { Problemas de conducta } \\
\text { Caídas frecuentes } \\
\text { Ataxia, distonía, disfagia, disartria } \\
\text { Convulsiones (locales y/o generalizadas) } \\
\text { Cataplexia } \\
\text { Mioclonía } \\
\text { *PSMV (habitualmente presente) }\end{array}$ \\
\hline $\begin{array}{l}\text { Adolescentes y Adultos } \\
>15 \text { años }\end{array}$ & $\begin{array}{l}\text { Organomegalia } \\
\text { (No siempre presente) }\end{array}$ & $\begin{array}{l}\text { Torpeza } \\
\text { Caídas frecuentes } \\
\text { Dificultad del aprendizaje o para trabajar } \\
\text { Síntomas neurológicos de progresión lenta } \\
\text { Ataxia, distonía, disfagia, disartria } \\
\text { Convulsiones (parciales y/o generalizadas) } \\
\text { Cataplexia } \\
\text { Demencia } \\
\text { Signos psiquiátricos } \\
\text { Mioclonía } \\
\text { *PSMV (casi siempre presente) }\end{array}$ \\
\hline
\end{tabular}

*PSMV=Parálisis Supranuclear de la Mirada Vertical. 
Esta enfermedad metabólica es difícil de diagnosticar porque presenta un fenotipo variable y esta categorizada según la edad de aparición de las manifestaciones clínicas en pre-perinatal, infantil-juvenil y adolescencia-adulto [7], como se detalla en el tabla 2.

\section{Resultados y discusión}

\subsection{Análisis cualitativo de la fluorescencia}

El análisis cualitativo de la intensidad de fluorescencia permitió identificar el diagnóstico bioquímico positivo asociado a NPC en estado aparente de homocigoto, observado por acumulo de LDL a nivel perinuclear en los fibroblastos, mediante tinción de Filipin (figura 1). En esta prueba bioquímica se activan los receptores de LDL como proceso inicial para continuar la ruta metabólica; alteraciones en la esterificación y regulación del colesterol producen depósito lisosomal, aunque también se almacena en el complejo de golgi y retículo endoplasmático [11, $12,13,14,15]$.
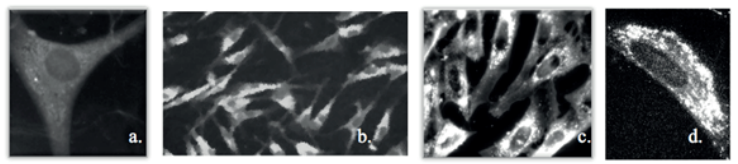

Figura 1. Diagnóstico bioquímico positivo por tinción de Filipin en fibroblastos cultivados en presencia de LDL por 24 horas. a) $100 \mathrm{X}$ Control Negativo: ausencia de intensidad de fluorescencia. b, c) 20X, d) 100X Alta intensidad de fluorescencia en forma de gránulos o numerosas inclusiones vesiculares a nivel perinuclear, evidencia de depósito de colesterol.

\subsection{Síntomas y edades de los pacientes evaluados}

La aparición de los síntomas en pacientes pediátricos y adultos con sospecha de NPC fue variable e independiente a la edad en relación al género (figura 2), el rango de edad osciló entre 0-25 años. Según nuestros datos la edad media al diagnóstico bioquímico positivo fue de 8.32 años, es decir; 9.9 para el género femenino y para el masculino 6.7 años.

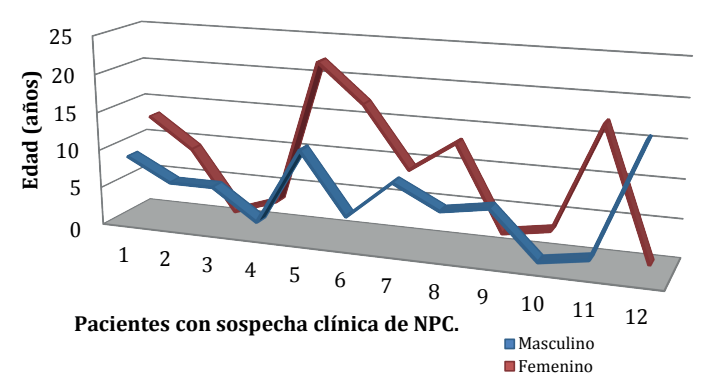

Figura 2. Diagnóstico bioquímico positivo por género y edad. Este patrón positivo se observó en pacientes pediátricos y adultos independientes a la edad y al género.

Los valores de la prueba $t$ (tabla 3 ), permitieron establecer que el promedio de edad de los pacientes con diagnóstico bioquímico positivo son iguales, que no existen diferencias significativas con un valor $p(p=0.2 ; p \geq 0.05 ; t=1.2)$, en referencia a la siguiente formulación:

Hipótesis nula $(\mathrm{Ho})$ : los promedios son significativamente iguales $(p \geq 0.05)$

Hipótesis alterna $(\mathrm{Ha})$ : los promedios son significativamente diferentes $(p<0.05)$.

Tabla 3. Valores prueba $t$, en relacion al género y edad de los pacientes con diagnóstico bioquímico positivo.

\begin{tabular}{lcc}
\hline Parámetro & Femenino & Masculino \\
\hline Recuento & 11 & 12 \\
Promedio & 9.9 & 6.7 \\
Varianza & 50.4 & 20.9 \\
Desviación Estándar & 7.1 & 4.5 \\
Coeficiente de Variación & $71.7 \%$ & $67.7 \%$ \\
Mínimo & 1 & 1 \\
Máximo & 22 & 17 \\
Rango & 21 & 16 \\
Cuartil Inferior & 3 & 3 \\
Cuartil Superior & 17 & 9 \\
Rango Intercuartílico & 14 & 6 \\
Sesgo Estandarizado & 0.3 & 1.3 \\
Curtosis Estandarizada & -0.8 & 0.7 \\
\hline
\end{tabular}

Para los pacientes con evidencia de depósito de colesterol a nivel perinuclear, el valor máximo de edad ( 25 años) para las mujeres fue 7 años mayor en relación a los hombres (18 años) ( figura 3). En consecuencia, las mujeres presentaron un rango de edad más amplio. Sin embargo, la prueba $t$ permite inferir que no hay diferencias estadísticamente significativas entre los promedios de las edades de los pacientes positivos para la prueba bioquímica. Los valores de curtosis permiten inferir una asimetría positiva con relación a la frecuencia de edad, dado que los valores están muy cercanos a la media. 


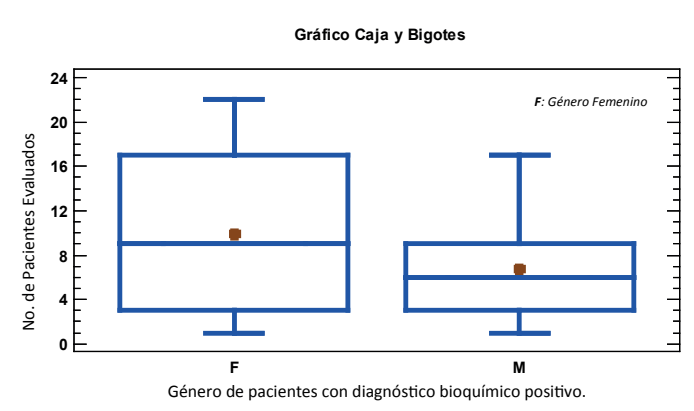

Figura 3. Edad por género de los pacientes con tinción de Filipin positiva. Para los pacientes con evidencia de depósito de colesterol a nivel perinuclear, el valor máximo de edad (25 años) para las mujeres fue 7 años mayor en relación a los hombres (18 años).

\subsection{Dificultades en los cultivos celulares}

Una de las limitaciones de la prueba bioquímica de tinción de Filipin se presentó en los cultivos de fibroblastos con resultado diagnóstico positivo, consistente en la dificultad para la adhesión y proliferación. Los fibroblastos cultivados de pacientes afectados presentaron cambios morfológicos inducidos por lisis celular como consecuencia del depósito de colesterol ( figura 4), condición no observada en los controles.

El Filipin reacciona con el colesterol no esterificado y detecta los derivados de éster colesterol, observados como partículas de variados tamaños a nivel intranuclear; este depósito de colesterol produce apoptosis celular $[16,17]$, este comportamiento permite inferir que el acumulo de colesterol es un mecanismo patogénico de muerte celular en pacientes con evidencia de depósito lisosomal. En este sentido, la alteración estructural es una de las consecuencias más relevantes de la enfermedad de NPC, porque confiere una morfología celular atípica, especialmente en las neuronas, lo que conlleva problemas de neurotransmisión [18]. Esto también, fue demostrado por los estudios de microarreglos (microarray) en fibroblastos de pacientes NPC, en los cuales, se concluye que estas células activan genes que inducen apoptosis [19]. En modelos animales con NPC, los hepatocitos de ratón también exhiben apoptosis, inflamación y fibrosis [20].
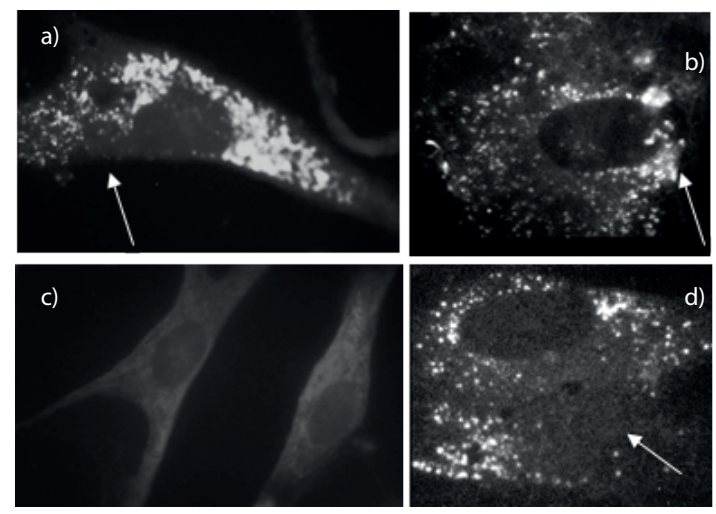

Figura 4. Lisis de Fibroblastos por depósito lisosomal de colesterol (100X) a, b, c) Fibroblastos de pacientes con diagnóstico bioquímico positivo; cultivados en presencia de LDL durante 24 horas. La flecha indica lisis celular por ruptura de membranas producido por depósito lisosomal de colesterol perinuclear. d) Fibroblastos normales.

La patogénesis de la enfermedad esta generada por alteración en las proteínas de los genes NPC1 y NPC2, involucradas en la exportación adecuada de colesterol y es posible que actúen de forma conjunta para regular el transporte lipídico intracelular y la homeostasis de colesterol. Mutaciones en estos genes generan un funcionamiento deficiente, lo que altera el transporte lipídico y conduce a la acumulación de colesterol en los lisosomas [6, 21]. La vitamina E también se acumula en los lisosomas y puede disminuir la capacidad antioxidante de la célula.

En este sentido, los pacientes con NPC presentan disminución en la capacidad antioxidativa y un incremento en productos derivados de la oxidación de colesterol, detectable en plasma por concentración de oxiesteroles; datos no incluidos en este estudio. Este daño oxidativo puede relacionarse con disfunción de las mitocondrias evento observado en muchas enfermedades neurodegenerativas [19]. Sepulveda y colaboradores proponen varios modelos matemáticos que pueden ser aplicables a nivel celular; para evaluar diferentes variables como la temperatura y la humedad relativa, que pueden influir en el daño oxidativo [22].

\section{Conclusiones}

La aplicación de LDL y tinción de Filipin en los fibroblastos in vitro, permitió el diagnóstico bioquímico positivo forma "clásica" asociada con NPC, mediante la intensidad de fluorescencia perinuclear, que facilitó visualizar el depósito lisosomal en los 
23 pacientes reportados. Sin embargo, el diagnóstico definitivo de NPC debe ser complementado por estudios moleculares para las mutaciones en los genes NPC1 y NPC2. Adicionalmente, estos datos deben correlacionarse con la historia clínica de los pacientes evaluados para evitar el subdiagnóstico, los falsos positivos e interpretación errónea con diagnósticos diferenciales que también evidencia fuerte fluorescencia pero sus manifestaciones clínicas no se asocian con NPC. La prueba $t$ permitió establecer que no existen diferencias estadísticamente significativas, entre las edades ni entre los géneros de los pacientes con diagnóstico bioquímico positivo.

\section{Agradecimientos}

A la Maestría en Ciencias Biológicas de la Universidad Pedagógica y Tecnológica de Colombia (UPTC), Centro de investigación GENETIX, $O N G$ Caminando Juntos, Marie T. Vanier PhD y a todas aquellas personas y entidades que brindaron su colaboración para el desarrollo de esta investigación.

\section{Referencias}

[1] X. Sun, D. L. Marks, D. L., Park, C. L. Wheatley, V. Puri, J. F. O’Brien, K. Snow. "Niemann-Pick C Variant Detection by Altered Sphingolipid Trafficking and Correlation with Mutations within a Specific Domain of NPC1". Am. J. Human Genet., vol. 68, no. 6, pp. 1361-1372, June, 2001.

[2] W. S. Garver, D. Jelinek, F. J. Meaney, J. Flynn, K. M. Pettit, G. Shepherd, G. A. Francis. "The National Niemann-Pick Type C1 Disease Database: correlation of lipid profiles, mutations, and biochemical phenotypes". J. Lipid Res., vol. 51, no. 2, pp. 406-415, February 2010.

[3] P. Marc. "Niemann-Pick Disease Type C. Synonym: Juvenile Niemann-Pick Disease". In Gene Reviews, R. A. Pagon, .M. P. Adam, H. H. Ardinger, S. E. Wallace, A. Amemiya, L. J. Bean, T. D. Bird, C. T. Fong, H. C. Mefford, R. J. Smith, K. Stephens, Ed. Universidad de Washington, Seatlle, 2012, pp. 1-30.

[4] M. T. Vanier, P. Latour. "Laboratory diagnosis of Niemann Pick disease type C: The filipin staining test". In Lysosomes and lisosomal diseases. Methods in Cell Biology, Ed. Frances
Platt and Nick Platt, Lyon, France, vol. 126, 2015, pp. 357-375.

[5] M. Patterson, C. Hendriksz, M. Walterfang, F. Sedel, M. T. Vanier, F. Wijburg. "Recommendations for the diagnosis and management of Niemann Pick disease type C: an update". Mol. Genet. Metab., vol. 106, no. 3, pp. pp. 357-375, July, 2012.

[6] E. Fernández, A. Ballart, C. Iturriaga, M. Lluch, J. Macias, M. T. Vanier, M. Pineda. "Identification of 25 new mutations in 40 unrelated Spanish Niemann Pick type C patients: genotypephenotype correlations". Clin. Genet., vol. 68, no. 3, pp. 245-254, September, 2005.

[7] W. Garver, G. Francis, D. Jelinek, G. Shepherd, J. Flynn, G. Castro, C. Walsh Vockley, Coppock, K. Pettit, R. Heidenreich, F. Meaney. The National Niemann Pick C1 database: report of clinical features and health problems. Am. J. Med. Genet. A, vol. 143A, no. 11, pp. 1204-1211, June 2007.

[8] F. A. Wijburg, F. Sedel, M. Pineda, C. J. Hendriksz, M. Fahey, M. Walterfang, M. C. Patterson, J. E. Wraith, S. A. Kolb. "Development of a suspicion index to aid diagnosis of Niemann Pick disease type C. Neurology, vol. 78, no. 20, pp. 1560-1567, May, 2012.

[9] E. Mengel, H. Klünemann, C. M. Lourenço, C. J. Hendriksz, F. Sedel, M. Walterfang, S. A. Kolb. "Niemann-Pick disease type $\mathrm{C}$ symptomatology: an expert based clinical description". Orphanet J. Rare Dis., vol. 8, no. 1, pp.166, October 2013.

[10] M.T. Vanier, D. A. Wenger, M. E. Comly, R. Rousson, R. O. Brady, P. G. Pentchev. "Niemann Pick disease group C: clinical variability and diagnosis based on defective cholesterol esterification: a collaborative study on 70 patients". Clin. Genet., vol. 33, no. 5, pp. 331-348, May, 1988.

[11] M. T. Vanier. Laboratory diagnosis of NiemannPick disease type C: "The filipin staining test". In Methods in Cell Biology, Lyon, France. Capitule 18, vol. 126, 2015.

[12] J. E. Vance, B. Karten B. "Niemann Pick C disease and mobilization of lysosomal cholesterol by cyclodextrin". J. Lipid Res., vol. 55, no. 8, pp. 1609-1621, March, 2014. 
[13] S. D. Goldman, J. P. Krise. "Niemann Pick C1 functions independently of Niemann Pick C2 in the initial stage of retrograde transport of membrane impermeable lysosomal cargo". J. Biol. Chem., vol. 285, no. 7, pp. 4983-4994, February, 2010.

[14] M. E. López, A. D. Klein, U. J. Dimbil, M. P. Scott. "Anatomically defined neuron-based rescue of neurodegenerative Niemann-Pick type C disorder". J. Neurosci., vol. 31, no. 12, pp. 4367-4378, March 2011.

[15] E. J. Blanchette-Mackie, N. K. Dwyer, L. M. Amende, H. S. Kruth, J. D. Butler, J. Sokol, M. E. Comly, M. T. Vanier, J. T. August, R. O. Brady. "Type-C Niemann-Pick disease: Lowdensity lipoprotein uptake is associated with premature cholesterol accumulation in the Golgi complex and excessive cholesterol storage in lysosomes". Proc. Natl. Acad. Sci. USA., vol. 85, no. 21, pp. 8022-8026, November, 1988.

[16] H. S. Kruth. "Histochemical detection of esterified cholesterol within human atherosclerotic lesions using the fluorescent probe filipin". Atherosclerosis, vol. 51, no. 2-3, pp. 281-292, May-June, 1984.

[17] H. S. Kruth. "Accumulation of unesterified cholesterol in limbal cornea and conjunctiva of rabbits fed a high-cholesterol diet. Detection with filipin". Atherosclerosis; vol. 63, no.1, pp: 1-6, January, 1987.
[18] F. A. Wijburg, F. Sedel, M. Pineda, C. J. Hendriksz, M. Fahey, M. Walterfang, M. C. Patterson, J. E. Wraith, S. A. Kolb. "Development of a suspicion index to aid diagnosis of NiemannPick disease type C". Neurology, vol. 78, no. 20, pp. 1560-1567, May, 2012.

[19] M. C. Vázquez, E. Balboa, A. R. Álvarez, S. Zanlungo. "Oxidative stress: a pathogenic mechanism for Niemann Pick type C disease". Oxid. Med. Cell Longev., vol. 2012, no. 2012, pp. 205-713, June, 2012.

[20] V. M. Rimkunas, M. J. Graham, R. M. Crooke, L. Liscum. "In vivo antisense oligonucleotide reduction of NPC1 expression as a novel mouse model for Niemann Pick type $\mathrm{C}$ associated liver disease". Hepatology, vol. 47, no. 5, pp. 15041512, May, 2008.

[21] A. Frolov, S. E. Zielinski, J. R. Crowley, N. Dudley-Rucker, J. E. Schaffer, D. S. Ory D. "NPC1 and NPC2 regulate cellular cholesterol homeostasis through generation of low density lipoprotein cholesterol derived oxysterols". J. Biol. Chem., vol. 278, no. 28, pp. 25517-25525, July, 2003.

[22] O. Sepulveda, Z. Suarez, M. Patarroyo, L. Canaria, S. Bautista, "Estudio del comportamiento e impacto de la climatología sobre el cultivo de la papa y del paso en la región central de Boyacá empleando los sistemas dinámicos", Ciencia en Desarrollo, vol. 6, no. 2, pp. 215224, 2015. 